\title{
Large-Scale Fluctuations of Pressure in Fluid Flow Through Porous Medium with Multiscale Log-Stable Permeability
}

\author{
Olga Soboleva \\ Institute of Computational Mathematics and Mathematical Geophysics, Novosibirsk \\ 630090, pr. Lavrentieva 6, Russia \\ olga@nmsf.sscc.ru
}

\begin{abstract}
In this paper, we consider subgrid modeling of a filtration flow of a fluid in a nonhomogeneous porous medium. An expression for the effective permeability coefficient for the large-scale component of the flow is derived. The permeability coefficient possesses a log-stable distribution. The obtained formulas are verified by the numerical modeling.
\end{abstract}

\section{Introduction}

Field and laboratory observational data imply that such parameters as permeability field and porosity have rather an irregular varying character. In this case small-scale details of permeability and porosity are unknown. The latter are taken into consideration in the statistical models [1],[2], [3],[5], using effective coefficients. In [2], [3], based on the ideas of the renormalized group (RG) by Wilson [4], the subgrid formulas for the effective permeability coefficient were derived, and the diffusion of the interface of liquids in their combined current in a scaleinvariant porous medium for log-normal permeability distributions was studied. The Landau-Lifshits formula for the effective permeability within a strict field RG was calculated in Teodorovich [5]. In particular, there are mentioned arguments from monograph [6], according to which the RG methods partially take into account higher orders of the perturbation theory and are to improve the accuracy of the formulas to be obtained. The same arguments are also applicable to the subgrid modeling. If a medium is assumed to satisfy the improved similarity hypothesis by Kolmogorov [2], [7] the RG equations take a very simple form. In this paper, the Wilson RG ideas are used for deriving the subgrid modeling formulas when solving problems of filtration in a multiscale porous medium with a log-stable distribution of permeability. The differential equations for obtaining effective constants were also derived for the media that do not satisfy the improved similarity hypothesis. The formulas obtained are verified by the numerical modeling.

\section{Statement of Problem}

For small Reynolds' numbers, the filtration velocity $\mathrm{v}$ and the pressure are connected by the Darcy law $\mathbf{v}=-\varepsilon(\mathbf{x}) \nabla p$, where $\varepsilon(\mathbf{x})$ is a random coordinate 
function - the permeability coefficient. An incompressible liquid flows through the medium. The incompressibility condition brings about the equation

$$
\nabla[\varepsilon(\mathbf{x}) \nabla p(\mathbf{x})]=0 .
$$

Let a permeability field be known. This means that at each point $\mathbf{x}$, the permeability is measured by pushing the liquid through a pattern of a very small size $l_{0} \mathrm{~A}$ random function of spatial coordinates $\varepsilon(\mathbf{x})$ is regarded as permeability limit at $l_{0} \rightarrow 0, \varepsilon(\mathbf{x})_{l_{0}} \rightarrow \varepsilon(\mathbf{x})$. In order to turn to a coarser grid $l_{1}$, it is impossible just to smooth the obtained field $\varepsilon(\mathbf{x})_{l_{0}}$ in the scale $l_{1}>l_{0}$. The field obtained is not a true permeability, which describes filtration in the domain of scales $\left(l_{1}, L\right)$, where $L$ is the largest scale. In order that permeability be defined on a coarser grid, measurements should be taken again by pushing the liquid through larger samples of the size $l_{1}$. The necessity of fulfilling this procedure is due to the fact that the permeability fluctuations from the limit $\left(l_{0}, l_{1}\right)$ have correlations with the pressure fluctuations induced by them. Similar to Kolmogorov [7], we consider the dimensionless field $\psi$ equal to the relation of permeabilities smoothed in two different scales $\left(l, l_{1}\right)$. This approach is described in detail in [2]. Let us denote by $\widetilde{\varepsilon}(\mathbf{x})_{l}$ the smoothed in scale $l$ the permeability $\varepsilon(\mathbf{x})_{l_{0}}$, then $\psi\left(\mathbf{x}, l, l_{1}\right)=\widetilde{\varepsilon}(\mathbf{x})_{l_{1}} / \widetilde{\varepsilon}(\mathbf{x})_{l}$. When $l_{1} \rightarrow l$, the field $\varphi(\mathbf{x}, l)=\left.\frac{d \psi(\mathbf{x}, l, l \lambda)}{d \lambda}\right|_{\lambda=1}$ is obtained that defines all statistical attributes of a porous medium. The relation obtained represents a differential equation, whose solution yields the permeability as function of the field $\varphi$

$$
\varepsilon(\mathbf{x})_{l_{0}}=\varepsilon_{0} \exp \left[-\int_{l_{0}}^{L} \varphi(\mathbf{x}, l) \frac{d l}{l}\right] .
$$

The permeability is assumed to have inhomogeneities of the scales $l$ from the interval $l_{0}<l<L$, and the field $\varphi$ is isotropic and homogeneous. The fields $\varphi(\mathbf{x}, l), \varphi\left(\mathbf{y}, l^{\prime}\right)$, with different scales for any $\mathbf{x}, \mathbf{y}$ are statistically independent. This hypothesis is generally assumed to be correct for different models and reflects the fact that the statistical dependence attenuates when fluctuations of scales differ in value [7]. The scale properties of a model are defined from the field $\varphi(\mathbf{x}, l)$. For the scale-invariant models, the condition $\varphi(\mathbf{x}, l) \rightarrow \varphi(K \mathbf{x}, K l)$. should be fulfilled. According to the theorem of sums of independent random fields [8] if the variance $\varphi(\mathbf{x}, l)$ is finite, then for large $l$. Integral in (2) tends to the field with a normal distribution. However, if the variance is infinite and there exists a non-degenerate, limiting distribution of a sum of random values, then such a distribution will be stable. For simplicity, the field $\varphi(\mathbf{x}, l)$ is assumed to have a stable distribution. With $L / l_{0} \gg 1$, it is impossible to calculate the pressure from equation (1) or if it is, then it demands large computer costs. Therefore, we pose the problem to obtain effective coefficients in equations for the large-scale filtration components. For the subgrid modeling we use ideas of the Wilson RG. 


\section{A Log-Stable Permeability}

The growth of irregularity, chaotic state and intermittence in the behavior of physical fields with increasing the scale of measuring made many researchers to reject the log-normal model and consider a general case of log-stable distributions. In [9], distributions of permeability fields and some statistical characteristics were obtained using the experimental borehole data. Also, it was shown that the permeability fields can have log-stable distributions. Stable distributions depend on the four parameters $\alpha, \beta, \mu, \sigma,[10]$. The parameter $\alpha$ is within the domain $0<\alpha \leq 2$, where $\alpha=2$ corresponds to the Gauss distribution. Statistical moments of order $m$ for $m \geq \alpha$ do not exist except for the case $\alpha=2$, for which all the statistical moments are determined. Thus, the variance is infinite for $\alpha<2$, and the mean value - for $\alpha<1$. For modeling the field $\varphi(\mathbf{x}, l)$, having a stable distribution law, the approach from [11] is used. At the points $\left(\mathbf{x}_{j}, l\right)$, the field $\varphi$ is defined by the sum of random independent values having stable distributions with the same parameters $\alpha, \beta, \mu=0, \sigma=1$ (the form $A[10])$ ):

$$
\varphi\left(\mathbf{x}_{j}, l\right)=\left(\frac{\Phi_{0}(l)}{2(\delta \tau \ln 2)^{\alpha-1}}\right)^{\frac{1}{\alpha}} a_{\mathbf{j i}}^{l} \zeta_{\mathbf{i}}^{l}+\varphi_{0}(l),
$$

where $l=2^{\tau}, \delta \tau$ - is a discretization step, the coefficients $a_{\mathrm{ji}}^{l}$ have a support of size $l^{3}$, depend only on the module of difference of indices ( in the sequel the index $j$ can be omitted ) $a_{\mathbf{j i}}^{l} \equiv a^{l}(|\mathbf{i}-\mathbf{j}|)$, and for all $l$ the condition $\sum_{k_{x}} \sum_{k_{y}} \sum_{k_{z}}\left(a_{k_{x} k_{y} k_{z}}^{l}\right)^{\alpha}=1$ holds. For $1 \leq \alpha \leq 2$, the thus constructed field $\varphi$ can be stable, homogeneous and isotropic in spatial variables [11]. If the coefficients $a_{\mathbf{j i}}^{l}$ satisfy the condition $a_{\mathbf{j i}}^{l} \equiv a^{l}\left(\frac{|\mathbf{i}-\mathbf{j}|}{l}\right)$ and the constants $\Phi_{0}(l), \varphi_{0}(l)$ are the same for all $l$, then the field $\varphi$ will be invariant with respect to the scale transformation. The mean of the field $\varphi$ exists and is equal to $\varphi_{0}(l)$. As for the second moments, they are infinite for $\alpha \neq 2$. This complicates carrying out the correlation analysis, applied, for example, in [2] and the approach used in [5]. Nevertheless, for an extreme point $\beta=1$ the second moments for the permeability field itself exist despite of the absence of variance of the field $\varphi$. This case is of interest, because it was experimentally verified [9]. As $l_{0}$ is a minimum scale, we can set $\varepsilon(\mathbf{x})=\varepsilon(\mathbf{x})_{l_{0}}$. Thus, the permeability field $\varepsilon(\mathbf{x})$ within the above-described model has the form:

$$
\varepsilon(\mathbf{x})=\varepsilon_{0} \exp \left[-\left(\ln 2 \sum_{\widehat{l_{0}}}^{\widehat{L}} \varphi\left(\mathbf{x}, \tau_{l}\right) \delta \tau\right)\right]
$$

where $L=2^{\widehat{L} \delta \tau}, l_{0}=2^{\widehat{l_{0}} \delta \tau}$, and the integral in formula (2) is replaced by the sum. For the calculation of moments of first and second orders we use formulas from [11] for $\left\langle e^{-b \zeta}\right\rangle$. For the correlation permeability function we have the estimation:

$$
\langle\varepsilon(\mathbf{x}) \varepsilon(\mathbf{x}+\mathbf{r})\rangle \simeq C \exp \left[2 \delta \tau \ln 2\left(-2^{\alpha-2} \sum_{\widehat{l}_{r}}^{\widehat{L}} \Phi_{0}(\widehat{l})\left[\cos \left(\frac{\pi \alpha}{2}\right)\right]^{-1}-\varphi_{0}(\widehat{l})\right)\right]
$$


where $r=2^{\widehat{l_{r}} \delta \tau}$. For the self-similar permeability

$$
\begin{aligned}
\langle\varepsilon(\mathbf{x}) \varepsilon(\mathbf{x}+\mathbf{r})\rangle & \simeq C \exp \left[2\left(-2^{\alpha-2} \Phi_{0}\left[\cos \left(\frac{\pi}{2} \alpha\right)\right]^{-1}-\varphi_{0}\right)(\ln L-\ln r)\right] \\
& \simeq C\left(\frac{L}{r}\right)^{-2\left(2^{\alpha-2} \Phi_{0}\left[\cos \left(\frac{\pi}{2} \alpha\right)\right]^{-1}+\varphi_{0}\right)}
\end{aligned}
$$

The constant $C$ is not universal, and the exponent for the self-similar permeability in (6) is universal and according to [12] can be measured.

\section{A Subgrid Model}

Let us divide the permeability function $\varepsilon(\mathbf{x})=\varepsilon(\mathbf{x})_{l_{0}}$ into two components with respect to the scale $l$. The large-scale component $\varepsilon(\mathbf{x}, l)$ is obtained by statistical averaging over all $\varphi\left(\mathbf{x}, l_{1}\right)$ with $l_{1}<l$, while the small-scale component is equal to: $\varepsilon^{\prime}(\mathbf{x})=\varepsilon(\mathbf{x})-\varepsilon(\mathbf{x}, l)$ :

$$
\begin{aligned}
& \varepsilon(\mathbf{x}, l)=\varepsilon_{0} \exp \left[-\int_{l}^{L} \varphi\left(\mathbf{x}, l_{1}\right) \frac{d l_{1}}{l_{1}}\right]\left\langle\exp \left[-\int_{l_{0}}^{l} \varphi\left(\mathbf{x}, l_{1}\right) \frac{d l_{1}}{l_{1}}\right]\right\rangle_{<} \\
& \varepsilon^{\prime}(\mathbf{x})=\varepsilon(\mathbf{x}, l)\left[\frac{\exp \left[-\int_{l_{0}}^{l} \varphi\left(\mathbf{x}, l_{1}\right) \frac{d l_{1}}{l_{1}}\right]}{\left\langle\exp \left[-\int_{l_{0}}^{l} \varphi\left(\mathbf{x}, l_{1}\right) \frac{d l_{1}}{l_{1}}\right]\right\rangle_{<}}-1\right],
\end{aligned}
$$

where $\left\langle><\right.$ means averaging over $\varphi\left(\mathbf{x}, l_{1}\right)$ from the small scale $l_{1}$. The largescale (on-grid) pressure component $p(\mathbf{x}, l)$ is obtained as averaged solution to equation (1), where the large-scale component (1) is fixed, while the small-scale component $\varepsilon^{\prime}$ is random, $p(\mathbf{x}, l)=<p(\mathbf{x})>_{<}$. The subgrid component is equal to $p^{\prime}=p(\mathbf{x})-p(\mathbf{x}, l)$. Let us substitute the expressions for $p(\mathbf{x}), \varepsilon(\mathbf{x})$ in equation (1) with averaging over the component $\varepsilon^{\prime}$ :

$$
\nabla\left[\varepsilon(\mathbf{x}, l) \nabla p(\mathbf{x}, l)+<\varepsilon^{\prime}(\mathbf{x}) \nabla p^{\prime}(\mathbf{x})>_{<}\right]=0 .
$$

The second term in equation 9) is unknown, but cannot be rejected without preliminary estimation, since the correlation between permeability and the pressure gradient can be essential [1], [5]. The choice of the form of the second term in (9) determines a subgrid model. In order that values of such an expression be estimated, we apply the perturbation theory. In the Wilson RG, the initial value of the scale $l$ is close to that of the least scale $l$. Subtracting (9) from (1) we obtain the subgrid equation for the pressure $p^{\prime}(\mathbf{x})$ :

$$
\nabla[\varepsilon(\mathbf{x}) \nabla p(\mathbf{x})]-\nabla\left[\varepsilon(\mathbf{x}, l) \nabla p(\mathbf{x}, l)+<\varepsilon^{\prime}(\mathbf{x}) \nabla p^{\prime}(\mathbf{x})>_{<}\right]=0 .
$$


Equation (10) is used for finding the pressure $p^{\prime}(\mathbf{x})$ and cannot be accurately solved. From equation (10), neglecting second order terms of smallness, obtain

$$
\Delta p^{\prime}(\mathbf{x})=-\frac{1}{\varepsilon(\mathbf{x}, l)} \nabla \varepsilon^{\prime}(\mathbf{x}) \nabla p(\mathbf{x}, l)
$$

According to the Wilson $\mathrm{RG}$, the values $\varepsilon(\mathbf{x}, l), p(\mathbf{x}, l)$ from the right-hand side equation (11) are considered to be known, their derivatives changing slower than $\varepsilon^{\prime}(\mathbf{x})$. This corresponds to the idea to obtain a correct on-grid equation in the large-scale limit. Therefore,

$$
p^{\prime}(\mathbf{x})=\frac{1}{4 \pi \varepsilon(\mathbf{x}, l)} \int \frac{1}{r} \nabla \varepsilon^{\prime}\left(\mathbf{x}^{\prime}\right) d \mathbf{x}^{\prime} \nabla p(\mathbf{x}, l),
$$

where $r=\left|\mathbf{x}-\mathbf{x}^{\prime}\right|$. we come to the expression for a subgrid term in equation (9) in the large-scale limit:

$$
\begin{aligned}
\left\langle\varepsilon^{\prime}(\mathbf{x}) \nabla p^{\prime}(\mathbf{x})\right\rangle & \approx \frac{1}{4 \pi D \varepsilon(\mathbf{x}, l)} \int \Delta \frac{1}{r}\left\langle\varepsilon^{\prime}(\mathbf{x}) \varepsilon^{\prime}\left(\mathbf{x}^{\prime}\right)\right\rangle d \mathbf{x}^{\prime} \nabla p(\mathbf{x}, l) \\
& \approx-\frac{1}{D \varepsilon(\mathbf{x}, l)}\left\langle\varepsilon^{\prime}(\mathbf{x}) \varepsilon^{\prime}(\mathbf{x})\right\rangle \nabla p(\mathbf{x}, l),
\end{aligned}
$$

where $D$ is a spatial dimension, here $D=3$. The model is similar to that mentioned in Landau and Lifshits [13], which is used for effective dielectric permeability of a mixture under simplifying assumptions of small fluctuations of their spatial scale. From (7), (8) and using formulas [11], keeping only first order terms we will have for $\varepsilon^{\prime}(\mathbf{x})$, obtain

$$
\left\langle\varepsilon^{\prime}(\mathbf{x}) \varepsilon^{\prime}(\mathbf{x})\right\rangle \approx \varepsilon^{2}(\mathbf{x}, l) \delta \tau \ln 2\left[\cos \left(\frac{\pi}{2} \alpha\right)\right]^{-1}\left(1-2^{\alpha-1}\right) \Phi_{0}\left(\tau_{\widehat{l}}\right)
$$

For the second term in (9) we have

$$
\left\langle\varepsilon^{\prime}(\mathbf{x}) \nabla p^{\prime}(\mathbf{x})\right\rangle_{<} \approx-\frac{\delta \tau \ln 2}{D}\left[\cos \left(\frac{\pi}{2} \alpha\right)\right]^{-1}\left(1-2^{\alpha-1}\right) \Phi_{0}\left(\tau_{\widehat{l}}\right) \varepsilon(\mathbf{x}, l) \nabla p(\mathbf{x}, l)
$$

Substituting (14) in to (9) and keeping only first order terms we find

$$
\nabla\left[\left(1-\delta \tau \ln 2\left(\Phi_{0} \frac{2\left(1-2^{\alpha-1}\right)+D}{2 D \cos \left(\frac{\pi}{2} \alpha\right)}+\varphi_{0}\right)\right) \varepsilon(\mathbf{x}, l) \nabla p(\mathbf{x}, l)\right]=0 .
$$

In the subgrid modeling, the effective permeability coefficient in the scale $l$ must correctly describe the solution to equation (1) within the scales $(l, L)$ and be calculated by a formula of the same form as $\varepsilon(\mathbf{x})_{l_{0}}$. Thus, the effective permeability is determined from

$$
\varepsilon(\mathbf{x})=\varepsilon_{0 l} \exp \left[-\left(\ln 2 \sum_{\widehat{l}}^{\widehat{L}} \varphi\left(\mathbf{x}, \tau_{\widehat{l}}\right) \delta \tau\right)\right] .
$$


From (15) for $\delta \tau \rightarrow 0$ we obtain that the constant $\varepsilon_{0 l}$ satisfies the equation

$$
\frac{d \ln \varepsilon_{0 l}}{d \ln l}=-\Phi_{0}(l) \frac{2\left(1-2^{\alpha-1}\right)+D}{2 D \cos \left(\frac{\pi}{2} \alpha\right)}-\varphi_{0}(l),\left.\quad \varepsilon_{0 l}\right|_{l=L}=\varepsilon_{00}
$$

For a self-similar permeability, the solution to equation (17) has the form

$$
\varepsilon_{0 l}=\varepsilon_{00}\left(\frac{l}{L}\right)^{\Phi_{0} \frac{2\left(1-2^{\alpha-1}\right)+D}{2 D \cos \left(\frac{\pi}{2} \alpha\right)}+\varphi_{0}},
$$

The constant $\varepsilon_{00}$ describes the permeability in the largest possible scale for $l=L$.

\section{$5 \quad$ Numerical Modeling}

Equation (1) is numerically solved in the cube with the edge $L_{0}$. On the sides of the cube $y=0, y=L_{0}$ we set the constant pressure $\left.p(x, y, z)\right|_{y=0}=p_{1}$, $\left.p(x, y, z)\right|_{y=L_{0}}=p_{2}, p_{1}>p_{2}$. The pressure on other sides of the cube is set by the linear dependence along $y: p=p_{1}+\left(p_{2}-p_{1}\right) / L_{0}$. . The major filtration flow is directed along the axis $y$. Here, dimensionless variables are used. The problem is solved in the unit cube, with a unit pressure jump and $\varepsilon_{0}=1$. The permeability field is simulated by (4), $256 \times 256 \times 256$ grid in spatial variables is used, the scale step being $\delta \tau=1, \tau_{l}=0, \ldots,-8$.. The coefficients $a_{\mathbf{j i}}^{l}$ were selected as

$$
a_{\mathbf{j i}}^{l}=\left(\frac{\sqrt{\alpha}}{2^{\tau_{l}} \sqrt{\pi}}\right)^{3 / \alpha} \exp \left(-\frac{\left(\mathbf{x}_{\mathbf{j}}-\mathbf{x}_{\mathbf{i}}\right)^{2}}{2^{2 \tau_{l}}}\right) .
$$

The field $\varphi(\mathbf{x}, \tau)$ is generated independent for each $\tau_{l}$. The common exponent in (4) is summed up over statistically independent layers. For an approximate solution, it is possible to use a certain limited number of layers. We have selected the number of layers so that the scale of the largest permeability fluctuations would allow us to approximately change probabilistic mean values averaged over space and the scale of the smallest fluctuations, so that the difference problem will approximate equation (1)sufficiently well, whose grid analog is solved by the iterative method. Independent random values $\zeta_{\mathbf{i}}^{l}$ in formula (4) were simulated using the algorithm and the program cited [14]. For self-similar media the constants $\Phi_{0}, \varphi_{0}$ can be selected from experimental data for natural porous media. According to [12], the exponent in (6) for some natural media varies within $0.25-0.3$.

Fig. 1 shows the lines of the self-similar permeability level in the mid-span section $z=1 / 2$ for $\alpha=2$, which corresponds to the log-normal permeability model and $\alpha=1,6, \beta=1$, and, respectively, to a stable model. The parameters $\Phi_{0}=0.3, \varphi_{0}=0$. A difference between the two models is distinct.

According to the procedure of deriving a subgrid formula, for its verification it is necessary to numerically solve the full problem and to fulfil the probabilistic 

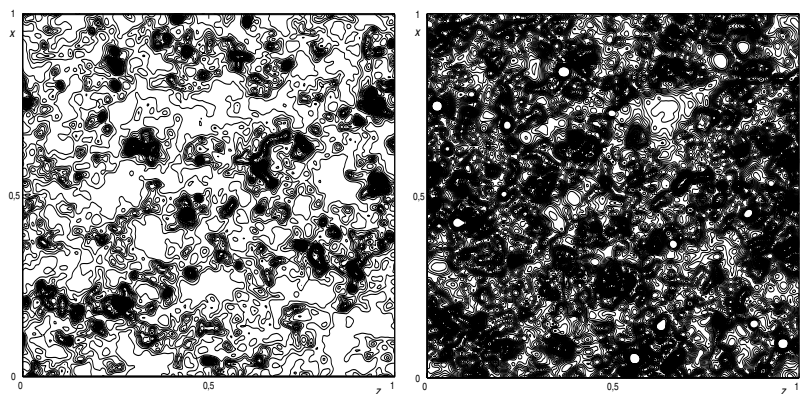

Fig. 1. The lines of levels in the mid-span section $z=1 / 2$ of the self-similar permeability for $\alpha=2$ (on the left), and $\alpha=1.6, \beta=1$ (on the right)

small-scale fluctuation averaging. As a result we obtain a subgrid term to be compared to a theoretical formula. This paper presents a more efficient version of such a verification that is based on the power dependence of the flow rate on the ratio of a maximum to a minimum scales in the ongrid domain when calculating permeability using (4) provided the contribution of a subgrid domain is not taken into account. The full flow rate of a liquid through a sample should coincide with the true one independent of the scale of the cut off $l$. The following formula is subject to verification:

$$
\left\langle\exp \left[-\left(\ln 2 \sum_{\widehat{l}}^{\widehat{L}} \varphi\left(\mathbf{x}_{j}, \tau_{l}\right) \delta \tau\right)\right] \nabla p\right\rangle=\frac{p_{2}-p_{1}}{y_{2}-y_{1}}\left(\frac{l}{L}\right)^{-\chi},
$$

where $\chi=\delta \tau \ln 2 \sum_{\widehat{l_{1}}=\widehat{l}}^{\widehat{L}}\left(\Phi_{0}\left(\tau_{\widehat{l_{1}}}\right) \frac{2\left(1-2^{\alpha-1}\right)+D}{2 D \cos \left(\frac{\pi}{2} \alpha\right)}+\varphi_{0}\left(\tau_{\widehat{l_{1}}}\right)\right)$, the probabilistic mean is replaced for the spatial averaging. This ergodic hypothesis was numerically verified. Then, using the numerical solution to (1), when the fluctuations are $\varepsilon_{-4}, \ldots, \varepsilon_{-6}$, we obtain the right-hand side of (20). Fig. 2 shows dependence
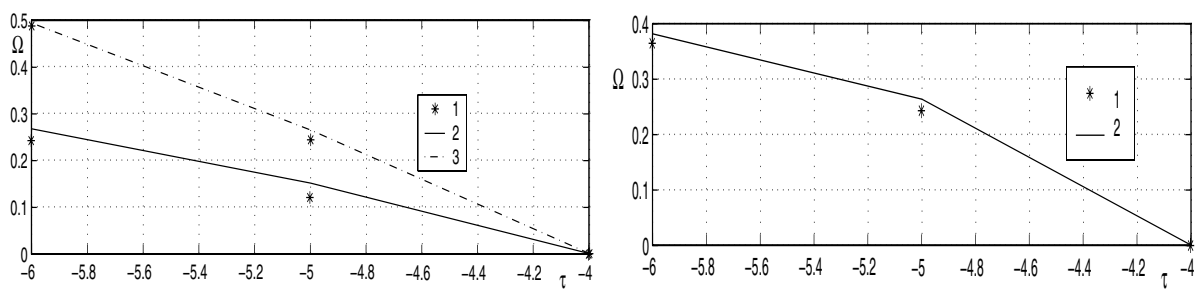

Fig. 2. The dependence of the logarithm of the flow rate $\Omega$ on the logarithm of the scale $\tau$ for $\alpha=1,6, \beta=1, \varphi_{0}=0$, the numerical simulation is marked stars. On the left - the self-similar model of permeability, 2 - for $\Phi_{0}=0.3,3$ - for $\Phi_{0}=0.6$. On the right - scale-invariance is absent, $\Phi_{0}(-4)=0.3, \Phi_{0}(-5)=0.6, \Phi_{0}(-6)=0.3$ 
of logarithm of the flow rate $\Omega\left(\tau_{\widehat{l}}\right)=\log _{2}\left\langle\exp \left[-\sum_{\widehat{l}}^{\widehat{L}} \varphi\left(\mathbf{x}_{j}, \tau_{\widehat{l}}\right) \delta \tau\right] \frac{\nabla p}{P_{2}-P_{1}}\right\rangle$ on the logarithm of scale $\tau$ for $\alpha=1,6, \beta=1, \varphi_{0}=0$. The results for a self-similar model of permeability are shown on the left Fig. 2. Line 2 corresponds to the value $\Phi_{0}=0.3$, line 3 corresponds to the value $\Phi_{0}=0.6$. The results obtained by numerical simulation are marked stars. On the right side there are shown the results for $\Phi_{0}(-4)=0.3, \Phi_{0}(-5)=0.6, \Phi_{0}(-6)=0.3$. In this case scale-invariance is absent. The numerical verification with the use of the spatial averaging is in good agreement with theoretical formulas. This paper was partly supported by the Integration Grant SB RAS No 61, and Grant RFBR 04-05-64415.

\section{References}

1. Shvidler, M. I.: Statistical hydrodynamics of porous media, Nedra, Moscow (1985) (in Russia)

2. Kuz'min G.A. and Soboleva O.N.: Subgrid modeling of filtration in porous selfsimilar media. J. Appl. Mech. and Tech. Phys. Vol. 43 (2002) 115-126

3. Kuz'min G.A. and Soboleva O.N.: Displacement of fluid in porous self-similar media. Physical Mesomechanics Vol. 5 (2002) 119-123

4. Wilson K. G. and Kogut J.: The renormalization group and the $\epsilon$-expansion. Physics Reports,12C(2) (1974) 75-199

5. Teodorovich E.V.: The renormalization group method in the problem of effective permeability of stochastically nonhomogeneous porous medium. (in Russian) JETP Vol. 122 (2002) 79-89

6. Bogolubov N. N. and Shirkov D. V.: Introduction of theory of quantum fields, Nauka, Moscow (1976)

7. Kolmogorov A. N.: A refinement of previous hypotheses concerning the local structure of turbulence in a viscous incompressible fluid at high Reynolds number. J. Fluid Mech. Vol. 13 (1962) 82-85

8. Gnedenko B.V. and Kolmogorov A.N.: Limit Distributions for Sums of Independent Random Variables. Addison-Wesley, Cambridge, MA., (1954)

9. Bouffadel M. C., S. Lu et al.: Multifractal scaling of the intrinsic permeability. Physics of the Earth and Planetary Interiors 36(11) (2000) 3211-3222

10. Zolotarev V.M.: One-dimensional Stable Distributions. Amer. Math. Soc., Providence, RI., (1986)

11. Samorodnitsky G. and Taqqu M. S. Stable non-Gaussian random processes. Chapman Hill., New York, London, (1994)

12. Sahimi M.: Flow phenomena in rocks: from continuum models, to fractals,percolation, cellular automata, and simulated annealing. Reviews of Modern Physics 65(4) (1993) 1393-1534

13. Landau L.D. and Lifshitz E.M.: Electrodynamics of Continuous Media. Pergamon Press, Oxford-Elmsford, New York, (1984)

14. Chambers J. M., Mallows C., Stuck B. W. A method for simulating stable random variables. Jornal of the American Statistical Association, 71(354) (1976) 340-344 